is quite different. The convection of light is given by the theory of relativity as a correction to the formulæ of classical dynamics. The observed shift of the interference bands is in perfect agreement with the new mechanics.

The Doppler effect is of interest in connexion with the experiments described. An application of the theory of relativity to the theory of the Doppler effect was made by Einstein. It is characteristic of the theory of relativity that this effect does not become zero when the motion of the source is at right angles to the direction of observation. According to theory, there remains a Doppler displacement of the spectral lines of extremely small amount. Einstein pointed out seventeen years ago that there was a possibility of observing this transverse effect by means of observations on canal rays. Experiments have been made on this subject, but different sources of error must be eliminated before the existence of this effect, which would be in harmony with the so-called dilatation of time, can be considered as proved.

\section{The Interrelation between Physics and Industrial Research.}

THE series of lectures which are being given under the auspices of the Institute of Physics have been concerned with the value of the physicist in modern industry. In his presidential address delivered at the annual general meeting on May 26, Sir Charles Parsons dealt with the converse influence which industry and industrial progress have exerted upon science, a beneficial influence which has not, perhaps, received sufficient recognition.

It is noteworthy, although not altogether surprising, that some of the greatest of the creators of the sciences of energy and thermodynamics were impelled by their engineering interests to take up their scientific work. In the case of James Watt, it was his interest in engineering which led him to his great work on the physical properties of steam. Joule began his work which led to the discovery of the mechanical equivalent of heat from attempts to solve an impossible engineering problem, namely, the supersession of the steam engine as a prime mover by an electromagnetic engine worked by the electrolytic consumption of zinc. The case of Carnot is a classic example of a similar procedure, and the birth of the modern science of thermodynamics was the result of a reconciliation, effected chiefly by Thomson, Clausius, and Rankine, of the discoveries of Joule and Carnot. A more recent example quoted was that of Lord Armstrong, who, though known chiefly as the introducer of hydraulic machinery and modern guns, was essentially a physicist in his methods of thought and investigation in engineering problems. His invention of the hydroelectric machine for generating hightension electricity arose from a chance observation of the emission of electric sparks from a tie bar in the roof of a boiler house.

In the field of invention proper, Sir Charles Parsons considered that the testing of a discovery or invention was probably the most important and difficult part of the work, and here a knowledge of physics was of inestimable value. Some interesting sidelights were thrown on the invention of the steam turbine. Some preliminary experiments were made with high-speed shafts and bearings, but in order to complete the data a small turbine coupled to a high-speed dynamo of primitive design was made ; the calculated stresses due to centrifugal force, the laws governing the flow of steam, and data from dynamos as approximately known at that time were taken into account. The constants for the flow of steam, the loss by friction in bearings at high surface speeds, the hysteresis and eddy current losses in armature core, conductors, and binding wire at abnormally high speeds were approximately investigated. Higher mathematics were not employed in this work, but were used much later to co-ordinate the accumulated data and forecast the effect of small improvements and refinements which have, in recent years, considerably increased the thermal efficiency of the turbine. The invention of the steam turbine reacted upon science by stimulating research in the dynamics of rotating shafts, the law of flow of saturated and superheated steam through jets, and the frictional resistances to flow through passages and over surfaces.

The address concluded with a consideration of the position in Great Britain of the scientific instrument industry. The industry, never a lucrative one, now snds itself, as a result of post-War conditions, in a position of extreme difficulty. Being small in magnitude out of all proportion to its importance, it is at a great disadvantage as compared with richer industries in facing times of depression. In the opinion of Sir Charles Parsons, perhaps a too-exclusive individualism, involving unnecessary overlapping and lack of interchange of knowledge among the various companies, may have been more pronounced than in some other lines of manufacture. At the same time there has grown up among physicists and others the practice of equipping their laboratories with instruments and materials largely imported from abroad. This has had a twofold injurious effect. It has reduced the home market and it has to some extent put the industry out of touch with the requirements of research workers and manufacturers. Sir Charles urged the users of scientific instruments to give more encouragement and sympathy to the home manufacturer, so as to enable him to provide himself with better equipment and a more skilled staff. Only thus will he be able to manufacture as cheaply and as well as the foreign manufacturer.

\section{University and Educational Intelligence.}

ABERYSTWYTH.-Applications are invited for the professorship of agriculture in the University College of Wales, vacant by the death of Prof. A. E. Jones. A knowledge of Welsh is desirable. Further particulars are obtainable from the General Secretary. The latest date for the receipt of applications is June $2 \mathrm{I}$.

Cambridge.-Prof. A. S. Eddington and Dr. P. A. MacMahon are to be appointed representatives of the University at the International mathematical congress at Toronto in August. Mr. A. Wood, Emmanuel College, has been re-appointed University lecturer in experimental physics. Mr. H. W. Florey has been elected to the John Lucas Walker Studentship.

The Council of the Senate has, on the suggestion of the Statutory Commission, proposed to the Senate a Statute to carry out the recommendation of the Royal Commission, to the effect that "no person shall be matriculated who has not passed or been exempted from an Examination to be recognised for the purpose by the University, except duly admitted Research Students and other persons or classes of persons whom the council of the Senate shall have approved for that purpose."

A proposal has been brought forward for an important change in Part II. of the Natural Sciences Tripos, namely, the introduction of the two subjects of biochemistry and pathology.

A Denman Baynes Studentship for research in NO. 2849 , VOL. I I 3$]$ 